\title{
EL DESARROLLO SOCIAL SOSTENIBLE, APLICADO EN LA EDUCACIÓN: MODELO PEDAGÓGICO DESDE LA SOCIOFORMACIÓN
}

\section{SUSTAINABLE SOCIAL DEVELOPMENT APPLIED TO EDUCATION: PEDAGOGICAL MODEL FROM SOCIO- EDUCATION}

\author{
Gabriela Lorena Abril Lucero ${ }^{1}$ \\ Diana Carolina García Ramos ${ }^{2}$ \\ Diana Carolina Abril Lucero ${ }^{3}$ \\ Fernanda Elizabeth Hidalgo Ortiz ${ }^{4}$
}

\author{
Recibido: 2021-05-12 / Revisado: 2021-06-20 / Aceptado: 2021-08-12 / Publicado: 2021-09-15
}

Forma sugerida de citar: Abril-Lucero, G. L., García-Ramos, D. C., Abril-Lucero, D. C., y Hidalgo-Ortiz, F. (2021) El desarrollo social sostenible, aplicado en la educación: Modelo pedagógico desde la socioformación. Retos de la Ciencia. 5(e). 76-86. https://doi.org/10.53877/rc.5.e.20210915.07

\section{RESUMEN}

La educación es uno de los principales medios para llegar a las personas con conocimientos para su crecimiento tanto académico como personal, por lo que resulta una vía pragmática para educar en sostenibilidad. Se busca mejorar hábitos y satisfacer necesidades que han sido causantes de problemas sociales que se presentan en la actualidad, sin comprometer los recursos a futuro; además busca un equilibrio entre el medio ambiente y el ser humano. Mediante la socioformación que contribuye a través de su modelo educativo a la resolución de problemas del contexto que originan una necesidad prioritaria, se puede aplicar un desarrollo social sostenible mediante la educación, que aplique alternativas de solución a los problemas detectados, que tengan impacto en mejorar las condiciones de vida de los seres humanos, obteniendo productividad. Este trabajo tuvo como objetivos describir los

\footnotetext{
1 Docente Investigadora en la Facultad de Ciencias Humanas y de la Educación de la Universidad Tecnológica Indoamérica. Miembro del grupo de Investigación, Desarrollo, Creatividad, Aprendizaje y Educación (DIDAC-PSI). Ecuador. E-mail: gabrielaabril@uti.edu.ec / ORCID: https://orcid.org/0000-0003-0233-6651

2 Docente Investigadora. Universidad Técnica de Ambato. Ecuador. E-mail: dc.garcia@uta.edu.ec / ORCID: https://orcid.org/0000-0002-6005-4532

${ }^{3}$ Docente en la Facultad de Arquitectura y Urbanismo. Universidad UTE. Ecuador. E-mail: diana.abril@ute.edu.ec / ORCID: https://orcid.org/0000-0003-1019-0891

4 Docente Unidad Educativa San Pío X. Ecuador. E-mail: ortiz@uepiox.edu.ec / ORCID: https://orcid.org/00000002-7841-0582
} 
beneficios de construir la sostenibilidad en la educación, identificar las características que utiliza el enfoque socioformativo y determinar las acciones prioritarias para fortalecer la sostenibilidad en diferentes áreas de producción. Se realizó una búsqueda bibliográfica documental de los últimos diez años sobre sostenibilidad y educación, de lo que se concluye que, mediante la educación se puede adquirir mejores hábitos de conducta dentro de la igualdad social, género, cuidado del medio ambiente y de uno mismo, prevaleciendo los derechos humanos sobre todas las cosas, para mejorar la calidad de vida.

Palabras clave: calidad de vida, desarrollo sostenible, educación, equilibrio, hábitos, problemas sociales, socioformación.

\section{ABSTRACT}

Education is one of the main means to reach people with knowledge for their academic and personal growth, which is why it is a pragmatic way to educate in sustainability. It seeks to improve habits and satisfy needs that have been the cause of social problems that arise today, without compromising future resources; It also seeks a balance between the environment and the human being. Through the socioformation that contributes through its educational model to the resolution of problems in the context that originate a priority need, a sustainable social development can be applied through education, which applies alternative solutions to the problems detected, which have an impact on improving the living conditions of human beings, obtaining productivity. This work aimed to describe the benefits of building sustainability in education, identify the characteristics used by the socioformative approach and determine the priority actions to strengthen sustainability in different areas of production. A documentary bibliographic search of the last ten years on sustainability and education was carried out, from which it is concluded that, through education, better habits of behavior can be acquired within social equality, gender, care of the environment and of oneself, prevailing human rights over all things, to improve the quality of life.

Keywords: balance, education, habits, quality of life, social problems, socioformation, sustainable development.

\section{INTRODUCCIÓN}

Desde hace mucho tiempo se ha hablado de sostenibilidad, pero no es hasta la actualidad, que se ha propagado de manera relevante en diferentes áreas tales como académicas, económicas y sociales, esto debido a que el impacto ocasionado al medio ambiente y a todo el contexto, ha presentado siniestros después de años de haberlo explotado para beneficio económico sin ningún cuidado alguno. Los diferentes efectos evidenciados en el medio ambiente y la vida han determinado un deterioro muchas veces irreversible, es por esto que de una manera urgente se ha tratado de incluir en la cotidianidad y en la vida productiva industrial, la sostenibilidad como una estrategia de proteger y salvaguardar la salud, el ambiente y la calidad de los seres vivos, con el fin de que la sostenibilidad se convierta en un estilo de vida.

Según Alaña et al. (2017) mencionan que el concepto de sostenibilidad hace referencia al "desarrollo que satisface las necesidades actuales sin comprometer la capacidad de las futuras generaciones de cubrir sus propias necesidades" (p. 93). La sostenibilidad, apuesta por un futuro en el que se consideren los derechos humanos, la salud y la economía con respeto al medio ambiente. Se hace énfasis en un equilibrio 
entre el cuidado al planeta, el crecimiento económico y el bienestar social. La sostenibilidad social hace posible que se mantenga un desarrollo vital en diferentes grupos sociales.

Es responsabilidad de los gobiernos y de la sociedad civil, lograr un futuro sostenible en la comunidad, existe una variedad de medios en donde se pueden aplicar estrategias para educar a estudiantes, a sus familias y a la sociedad en general en cuanto a cumplir con acciones sostenibles. La educación es esencial para lograr un futuro sostenible en las generaciones, en estudiantes, profesionales y próximos líderes que estarán preparados con conocimientos y actitudes para crear una vida con mucha más sostenibilidad (UNESCO, 2012).

Los objetivos del desarrollo sostenible son integrados, dependen de las realidades de cada país, respetando a sus capacidades y prioridades. Los gobernantes incorporarán a sus planificaciones las acciones que estén encaminadas a cumplir con los objetivos sostenibles, tales como trabajar en la trasformación en educación género y desigualdad; salud, bienestar y demografía; descarbonización de la energía e industria sostenible; modelo alimentario, tierra, agua y océanos; ciudades y comunidades sostenibles; revolución digital para el desarrollo sostenible (Gorospe et al., 2021).

Las naciones se enfrentan a diversos desafíos para cumplir con dichos hábitos, debido a que se debe considerar que los países menos desarrollados, en vías de desarrollo o sin litoral, atravesarán mayores dificultades (ONU, 2015).

En la educación a través del modelo pedagógico de la socioformación, se encuentra un desarrollo integral de las personas en varias áreas y ámbitos a través de la ética y de una reflexión constante, utilizando como apoyo la tecnología y la información para sentar una base de una sociedad del conocimiento. Además de que su carácter de aplicación en la realidad es a través de la solución de problemas y retos del contexto mediante estrategias acordes a la necesidad que se esté buscando atender, es una forma de educar en la sostenibilidad, resolviendo problemas de la cotidianidad mejorando la calidad de vida y el cuidado al medio ambiente y el desarrollo productivo que busca la sostenibilidad dentro de un reto contextual, mediante el pensamiento complejo (Lara et al., 2019). Este trabajo tiene como objetivos describir los beneficios de construir la sostenibilidad en la educación, identificar las características que utiliza el enfoque socioformativo, y determinar las acciones prioritarias para fortalecer la sostenibilidad en diferentes áreas de producción.

\section{DESARROLLO}

\section{Desarrollo social sostenible en la educación}

El sistema educativo está encaminado a hacer un aporte activo a la sociedad, en pro de un desarrollo sostenible, que priorice la justicia social y viabilidad ecológica. Las instituciones educativas, deben implementar esfuerzos para que sus procesos y operaciones escolares sean ecológicas, dando de esta manera en todo su actuar, un buen ejemplo a los jóvenes e incentivarlos a ser sostenibles. Educar y formar en sostenibilidad significa conciliar la teoría (enseñanza) con la práctica (vida escolar).

Las conductas de los estudiantes deben estar influenciadas no solo por las lecciones académicas de las asignaturas, sino también por la experiencia y observación cotidiana de su entorno escolar, viviendo en sostenibilidad. Para esto, las instituciones educativas, deben esforzarse por resolver sus propios déficits 
ecológicos, de modo que el conocimiento y las acciones que se empleen, estén al mismo nivel. Varias iniciativas podrían tener impacto en algunas áreas, en este ecosistema escolar que podrían servir de ejemplo a los estudiantes, otorgando una oportunidad de cambio cultural y de aprendizaje a través de resolución problemas en casos reales (Fundament, 2021).

El desarrollo sostenible, influye a toda la comunidad educativa, a que se enfrenten frecuentemente a nuevas intenciones de innovación, solucionando necesidades y es ahí un buen momento para hacerlo con hábitos de sostenibilidad.

Pero ¿qué es la sostenibilidad en la educación? Desde los años 90, la educación para el desarrollo sostenible avanzó de manera creciente, en el ámbito educativo formal y no formal, aunque no generó los cambios esperados en cuanto a las conductas y comportamientos de los ciudadanos, ni relevancia en la política y sociedad. Ocurrió al contrario una paradoja en la ciudadanía, tenía acceso suficiente a gran cantidad de información, pero seguían actuando como si los problemas no existieran (Gorospe et al., 2021).

Esto puede ser causal de que la prioridad de la educación para la sostenibilidad en las políticas y las instituciones educativas se ha quedado sólo en teoría, sin avanzar al carácter reproductivo de las estrategias de educación: el desarrollo sostenible necesita un cambio de paradigma sobre el modelo de crecimiento económico, dinámicas sociales y políticas (Fundament, 2021).

Las instituciones educativas, más allá de la transmisión de conocimientos, no cuestionan las conductas de dominancia con la naturaleza, el bienestar, los roles de género, la participación social y política y la dimensión sistémica de la emergencia climática, por lo que el cambio realmente es imperceptible. Sin entornos de aprendizaje significativo, será difícil ese cambio en las mentes a los cambios colectivos y sistémicos.

A través de la educación, se puede capacitar para actuar y desenvolverse en un entorno complejo, con cambios continuos en un contexto donde existen diversas narrativas individuales, con una creciente diversidad social y en el que los ecosistemas sociales y naturales en los que las personas interactúan están expuestas a una crisis sistémica de sostenibilidad. Para lograr cambios, alcanzar metas, la educación apuesta por el aprendizaje por competencias como estrategia para preparar a sus estudiantes para la vida, que puedan manejarse con autonomía y desarrollarse en la sociedad, que se desenvuelvan solos con hábitos sostenibles tanto en temas de la vida humana y natural, como en la productividad (Gorospe et al., 2021).

\section{Beneficios de construir la sostenibilidad en la educación}

Los beneficios de construir la sostenibilidad en la educación se evidencian en los primeros años de escolaridad, esto en virtud que las nuevas generaciones podrían tomar mejor conciencia desde la infancia al estar educados en sostenibilidad. La educación en la primera infancia brinda mejores resultados tanto cognitivos como conductuales, dentro y fuera del ámbito académico (UNESCO, 2015).

Un individuo durante su formación académica, que ha sido expuesto a información sobre los impactos de las inadecuadas actividades del hombre, puede producir en él cambios positivos en su comportamiento y así mejorar su calidad de vida (EQI, 2016). Esto respetando el equilibrio entre el medio ambiente, estilo de vida saludable, la equidad de género, la igualdad, la inclusión y en la producción de una economía sin afectación a la tierra en la que vivimos. Los beneficios de la sostenibilidad son la buena relación entre las personas, respeto de los derechos 
humanos sobre todas las cosas, el cuidado al medio ambiente y a la biodiversidad (EQI, 2016).

\section{Modelo pedagógico desde la socioformación}

El campo laboral demanda trabajadores emprendedores, con capacidad de responder a objetivos claros, con una visión de compromiso y con conocimientos pragmáticos basados en la ética, para que trabajen de manera colaborativa en la resolución de los problemas en el contexto local. Por esto se necesita formar a los ciudadanos para la sociedad del conocimiento (Tobón et al., 2015).

Esta necesidad requirió nuevos enfoques y modelos educativos, que respondan integralmente a los retos del nuevo tipo de sociedad que se busca crear.

Es así que, Tobón en el 2002 propuso un enfoque educativo que implicaba el espíritu humanista, empleando el pensamiento complejo como base, ubicando al ser humano como protagonista para abordar problemáticas reales que la misma sociedad tiene como preocupación, utilizando las tecnologías de una manera adecuada, con valores para formar una sociedad justa, consciente de su realidad y con miras de un mundo mejor.

Con esto nace el enfoque socioformativo, con una formación crítica, reflexiva, donde predominen los valores, la equidad de género, el respeto a la naturaleza, como cualidades principales (Prado, 2018).

\section{Características del enfoque socioformativo}

Según Lara et al. (2019) refieren que la socioformación es un enfoque educativo, cuya característica es buscar la formación integral del estudiante, con una visión del ser humano en proceso de realización, comprometido con pertenecer a una sociedad con mejores hábitos de vida en su contexto local. Este enfoque se ha manejado y aplicado en el ámbito académico superior, debido a que está acorde con las tendencias de sostenibilidad e innovaciones actuales. Además, busca contribuir a un mejor y adecuado desarrollo de la sociedad, partiendo desde el conocimiento dentro de la academia, con el propósito de generar el desarrollo social sostenible, aspecto que muy pocos enfoques educativos abordan.

Otra de las características de este enfoque, es que articula en su forma de operar a las cuatro funciones sustantivas de la educación de calidad superior, la docencia, investigación, vinculación con la sociedad y gestión administrativa, aplicadas en la multi, inter y transdisciplinariedad. En su base teórica utiliza mediante el logro de retos con colaboración, pensamiento complejo, emprendimiento, gestión del conocimiento y proyecto ético de vida, aparte que promueve la investigación de impacto acorde con las necesidades de la comunidad.

La socioformación, además se caracteriza por prevalecer el valor del individuo, no por la cantidad de conocimientos que adquiera, sino, en aplicar estos saberes para resolver problemas, a través del trabajo colaborativo, persiguiendo un meta común, alcanzando ganancias tanto individuales, sociales y ambientales, generando resultados a partir de la participación de los involucrados (Arreola et al., 2019).

La retroalimentación continua es otra característica de este enfoque educativo, que hace referencia a que el estudiante y el maestro puedan tener la oportunidad de identificar y reconocer lo que se enseñó y se aprendió, dándole claridad y solidez al desarrollo de competencias, utilizando la valoración de estas competencias alcanzadas a través de la autoevaluación, coevaluación y heteroevaluación, asumiendo cualquier error que pueda haberse dado, como una oportunidad de mejora y crecimiento. 
Por todas estas características, la socioformación se emplea para contribuir con el desarrollo social sostenible, procurando alcanzar niveles cada vez más altos en la calidad de vida, la sustentabilidad económica, convivencia y cuidado del ambiente y de la biodiversidad. La socioformación consiste en formar personas para la sociedad del conocimiento, mediante la resolución de problemas con participación colaborativa y empleo adecuado de las TICs (Tobón et al., 2015).

"Se diferencia de otros enfoques pedagógicos, por su carácter de aplicación en la realidad, abordando problemas del contexto dado que es aplicable en una institución educativa, una empresa, ámbitos comunitarios y que abordan problemáticas de acuerdo con su naturaleza" (Prado, 2018, p. 57).

\section{Acciones prioritarias para fortalecer la sostenibilidad en diferentes áreas de producción}

La contaminación que genera la producción debe disminuir notablemente. Evitar diseñar productos, actividades o servicios, que sean desechables ayudará a cumplir este objetivo, para esto los productos deben ser de larga duración con materia prima de calidad y crear un sistema circular de creación, reparación y reutilización.

Una acción prioritaria para fortalecer la sostenibilidad dentro de la producción también está en obtener las certificaciones sostenibles a cualquier tipo de producto 0 servicio que se esté entregando, esto se refiere a tener la certeza de la trazabilidad que confirme el proceso dentro de la sostenibilidad detrás de un producto, esto para garantía del consumidor (Abril, 2021). Finalmente, la sostenibilidad incentiva a concientizar que la gente se preocupe por su salud, a través de un mejor estilo de vida, sin dejar de lado el cuidado del planeta mediante la práctica de hábitos de consumo prácticos y renovables.

La economía circular es otra de las acciones prioritarias para la sostenibilidad, se encarga de reconstruir y regenerar los productos, con los mismos componentes y materiales. Es un proceso cíclico de desarrollo positivo de manera continua, que conserva y aumenta el capital natural, minimiza los riesgos del sistema y optimiza los rendimientos de los recursos, gestionando cantidades finitas y flujos renovables. Funciona de manera efectiva a cualquier escala (Cerdá y Khalilova, 2015).

\section{Dimensiones y principios de la educación para la sostenibilidad}

Figura 1:

Principios de la sostenibilidad

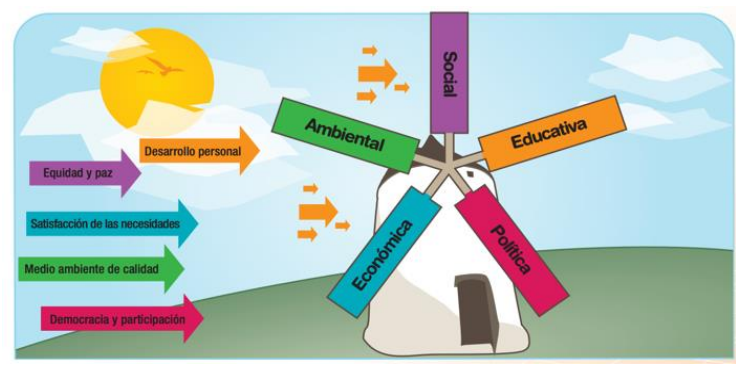

Fuente: Martínez (2015)

Las dimensiones de la sostenibilidad según Artara (2001) son económica, social y ecológica. La dimensión económica, ofrecería de forma ilimitada los recursos físicos y materiales para el desarrollo y crecimiento de los países, esto se ha tratado de controlar, cuidar y preservar los recursos naturales. La dimensión social, se refiere al 
poder que ejercen los seres humanos sobre el medio ambiente y contexto en general. $Y$ finalmente la dimensión ecológica, que habla de la economía circular, que se produzca un cierre de los ciclos, utilizando solamente recursos y energías renovables, evitando producir residuos, que regresaría a la misma naturaleza.

Las Naciones Unidas de la Educación para el Desarrollo Sostenible (2005-2014), tuvo como objetivo universal, asociar todos los valores que pertenecen al desarrollo sostenible en todas las facetas y procesos del aprendizaje, con vistas a motivar los cambios de conducta indispensables para lograr una sociedad sostenible y justa para todos (UNESCO, 2021).

En una sociedad, se evidencian problemas severos que se han perpetuado como problemas sociales a través del tiempo. Se puede mencionar tales como, problemas ambientales, tecnológicos, económicos, políticos, socioculturales, entre otros; conflictos que interaccionan entre sí. La educación para la sostenibilidad otorga las herramientas y pautas de acción, para comprender la relación entre los elementos que dan paso al origen del problema como al desarrollo sostenible de solución. Se tiene que aplicar como estrategia el diagnóstico, así como la propuesta de solución. Es así como, el desarrollo sostenible, es un conjunto de pasos que nos conduce a llegar a una meta y sólo se avanza si se aplican estrategias, técnicas y programas. Al ser un proceso paulatino social, está ligada directamente a las formas de comportamiento y conducta humana, que está regida por los valores que este posea. El aprendizaje se evidenciará en las conductas de la gente, en la construcción de hábitos de consumo.

La educación para la sostenibilidad pasa a ser una estrategia fundamental en este ámbito de solución o al menos de reducción de los problemas sociales (Martínez, 2015).

El desarrollo de un pueblo se mide por la economía de este lugar, por lo que, si se habla de desarrollo sostenible, implica observar aspectos que van mucho más allá de lo económico y material, sino aspectos además sociales y ambientales. Solano (2007) refiere que una sociedad que busque transformarse tiene que buscar cómo es que el respeto de la realidad suya y de los demás, generando condiciones para que los ciudadanos puedan crecer y progresar tanto a nivel humano como profesional, y de esta manera reconocer nuestro papel activo en la sociedad haciendo que nuestras tareas sean cumplidas en beneficio propio y colectivo, cuidando siempre por sobre todas las cosas el ambiente que nos ampara a todos como un grupo de personas de un mismo sector geográfico.

Es por esto por lo que, es importante entender y aceptar la propia situación económica o social tanto de abundancia o de escasez y que ninguna de estas posiciones otorgue el derecho a vulnerar los derechos de los demás. "Todo esto se debe realizar respetando, generando y fortaleciendo las necesarias capacidades humanas para ser partícipes de estos procesos. Sólo una sociedad que comprenda estos aspectos y trabaje en ellos está en el verdadero camino hacia su desarrollo" (Solano, 2007, p. 16). Es la clave en la búsqueda de mejorar el mundo en el que vivimos.

Es posible identificar procesos dentro de la insostenibilidad y la sostenibilidad. Las ciencias brindan instrumentos de evaluación y monitoreo, la sostenibilidad es un objetivo de políticas y se maneja dentro de una visión de ética de responsabilidad.

\section{Técnicas pedagógicas para la educación para la sostenibilidad}

El desarrollo sostenible debe adecuarse al entorno y a los contextos del medio local se logra al transmitir e interiorizar estos hábitos de vida a través de la educación 
para el desarrollo sostenible. La tarea de aprender a ser sostenibles se da con la educación, se debe instaurar el hábito de seguir aprendiendo constantemente a vivir una vida sostenible. $Y$ esto se puede otorgar con habilidades a las personas que tengan los conocimientos amplios y adecuados en ciencias humanas, ciencias naturales y ciencias sociales, así como en otras áreas no menos importantes como la economía (Pérez, 2018).

La educación para la sostenibilidad necesita hacer un diagnóstico de reconocimiento, de la crisis ecológica global, crisis de valores, ideas, perspectiva y conocimiento, con el objetivo de plantear habilidades y estrategias de solución en el ámbito académico (Sáenz y De Santiago, 2017).

Las habilidades y estrategias que se proponen para la educación según la UNESCO son comunicar de forma clara, efectiva, oral y/o escrita, trabajar en base al sistema contextual y el tiempo real, trabajar en conjunto, con perspectivas para comprender el punto de vista de todos, actuar en base a los valores culturales, sensibilizar la necesidad, aplicar conocimiento y a la acción para desarrollar alternativas de solución con ética y estética.

La educación para el desarrollo sostenible fomenta que los estudiantes se cuestionen, analicen, tengan un pensamiento crítico. Los escenarios para estimular este tipo de acciones suelen ser en artes como el teatro, la música, el diseño, el dibujo para estimular la imaginación y creatividad en alternativas de solución para el futuro. Con una visión de ser un miembro activo en la comunidad.

En el sistema académico se han implementado la discusión en clase como técnica pedagógica, en donde se realizan aportes críticos desde simulaciones en las que se instaura el aprendizaje a través de la discusión en una necesidad contextual; análisis de temas explorando los orígenes de problemas sociales y narración de historias, esta técnica resulta más atractiva en generaciones más jóvenes. Las actividades son dinámicas, adaptables y modificables según las necesidades del contexto, para lograr que la educación cuente con los principios básicos para el desarrollo de la sostenibilidad (Pérez, 2018).

\section{El desarrollo sostenible en la educación}

Según la UNESCO (2015) el objetivo de desarrollo sostenible es el de "garantizar una educación inclusiva, equitativa y de calidad y promover las oportunidades de aprendizaje durante toda la vida para todos", para lograr con esto se necesita que los gobiernos locales mantengan compromisos y alianzas que aseguren cumplir con el objetivo.

Todos los objetivos sostenibles necesitan de la educación para sensibilizar a las personas sobre los valores, información y responsabilidades que permitieran vivir en un ambiente más digno. En la actualidad hay 750 millones de adultos analfabetos, una de las causas que imposibilita el desarrollo social (UNESCO, 2021).

El desarrollo sostenible, educación y sostenibilidad pretenden resolver la falta de civilización actual. La educación superior con las universidades son parte de los protagonistas del desarrollo sostenible, porque es un medio de enseñanza. La universidad como entidad docente y de investigación es el principal agente de cambio social, mantiene el compromiso de generar soluciones a los problemas que impiden la sostenibilidad y a su vez capacitar a los seres humanos para que se logre ese cambio (Murillo, 2017).

La educación tiene la misión de potenciar los talentos y capacidades personales para que cada uno se responsabilice de sí mismo (Delors, 2001), sin embargo, esto dependerá de cada estudiante y de las características de personalidad más los 
factores culturales y redes de apoyo que posean. A través de la educación se alcanzan competencias les ayudaran a desenvolvernos en la sociedad. Un país con un alto nivel de desarrollo humano necesita el acceso a la educación en igualdad de condiciones y oportunidades para todos sus habitantes (Pérez, 2020).

En cuanto a la educación superior y sostenibilidad, los principios rectores según la UNESCO son que la educación es base de la realización humana, la paz, el desarrollo sostenible, el crecimiento económico, un trabajo digno, la igualdad entre los sexos y la ciudadanía mundial responsable. Además, la educación es una base en la reducción de las desigualdades y la pobreza, pues ofrece las condiciones y genera las posibilidades de existencia de sociedades mejores y más sostenibles (Gonzalo, 2017).

Para lograr el desarrollo sostenible desde la igualdad de oportunidades, el mismo organismo refiere que se debe tener un acceso equitativo a una educación de calidad para todos, niños, jóvenes y adultos, y se deberán mejorar las posibilidades de adquirir conocimientos y competencias para el desarrollo sostenible, la ciudadanía mundial y el mundo del trabajo (UNESCO, 2014).

Para reestructurar la educación superior hacia la sostenibilidad y aplicar en la práctica el modelo de fortalezas, es necesario revisar el plan de estudio para que este se encuentre amparado en los aportes que exige la sostenibilidad. Este análisis del currículo permitirá reorientar el plan de estudio, para hablar de temas de sostenibilidad. La realidad actual de muchos planes de estudio, si bien suelen incluir conocimientos y habilidades, los valores y perspectivas no forman parte de estos (ONU, 2012).

Es urgente la importancia de incorporar en la malla curricular de las diferentes carreras de las entidades de educación superior, módulos formativos que se relacionen con el medio ambiente, de tal forma que se haga efectivo el desarrollo sostenible. A través de la educación, se puede utilizar como instrumento para la reflexión y concientización, no sólo individual sino a nivel social, por lo que el conjunto de personas educadas en valores en común, hacen posible con el tiempo cambios sociales y comunitarios. La educación para el desarrollo sostenible requiere de un nuevo paradigma educativo que responda a los cambios sociales, económicos, culturales, políticos y axiológicos que permitan cumplir con los objetivos de sostenibilidad.

\section{CONCLUSIONES}

Educar dentro de la sostenibilidad, permite tomar una mejor conciencia desde tempranas edades para modificar hábitos y conductas para el cuidado de uno mismo, de los demás y del planeta, esto con visión de construir una mejor calidad de vida. Es importante considerar las características individuales de los estudiantes con el objetivo de formular estrategias que permitan llegar a todos con el conocimiento en sostenibilidad.

La socioformación se caracteriza por brindar una educación integral, con una propuesta interdisciplinaria, basándose en el servicio para resolver necesidades cotidianas reales del contexto, partiendo desde el conocimiento, con el propósito de generar un desarrollo social sostenible, es necesario capacitar a los docentes sobre sostenibilidad quienes son los principales conductores de conocimiento.

Las acciones prioritarias para fortalecer la sostenibilidad se basan en toda acción que esté encaminada a proporcionar una mejor calidad de vida, concientizando a la 
gente que se preocupe por su salud, generando mejores hábitos y cuidando del medio ambiente, responsabilizándose de verificar los procesos de producción de los productos o servicios que utilizamos, para exigir que se cumplan con la trazabilidad sostenible.

\section{REFERENCIAS BIBLIOGRÁFICAS}

Abril, C. (2021). Abril Diseño Sostenible. (G. Abril, Entrevistador).

Alaña, T., Capa, L., y Sotomayor, J. (2017). Desarrollo Sostenible. Universidad y sociedad, 91-99.

Artara, M. (2001). Teoría de las tres dimensiones de desarrollo sostenible. Ecosistemas. Revista ecológica y medio ambiente, 33(10). file://Users/gabriela/Downloads/614-Texto\%20del\%20art\%C3\%ADculo-11581-10-20120930.pdf.

Cerdá, E., y Khalilova, A. (2015). Economía Circular. Economía Circular, Estrategia y Competitividad Empresarial, 401, 11-20.

Delors, J. (2001), La educación encierra un tesoro, Ediciones Unesco, París.

Escuela de organización industrial, EQI. (2016). La educación para el desarrollo sostenible. Obtenido de Master Executive en Desarrollo sostenible y Responsabilidad corporativa.

Fundament. (2021). La sostenibilidad como camino hacia la educación holística. Obtenido de Fórmulas hacia la educación sostenible: https://www.fundament.es/escuelasostenible/?gclid=CjwKCAjw1JeJBhB9EiwAV612yyi8SkbJe4GlyPBh81LBUu1 mGGUuBg5TdokoGJMUO6YHUh0YEMASHBoC7LQQAvD_BwE.

Gonzalo, V., Sobrino, R., Benítez, L., y Coronado, A. (2017). Revisión sistemática sobre competencias en desarrollo sostenible en educación superior. Revista iberoamericana de educación.

Gorospe, B., Gehrig, M., Jiménez, M., y Rosado, I. (2021). Aprendiendo a bailar con el sistema. Madrid: Global Challenge.

Lara, S., Lara, D., y Tapia, F. (2019). Camino al éxito. Modelo educativo, pedagógico y curricular. El conejo.

Martínez, J. (2015). Ingurugela - Centro de Educación e Investigación Didáctico Ambiental. Obtenido de Educación para la sostenibilidad: https://www.unescoetxea.org/ext/manual_EDS/pdf/01_educacion_castellano. pdf

Murillo, B. (2017), Desarrollo sostenible y educación superior en un mundo global, V. 17, enero-abril 2017, Centro de Altos Estudios Universitarios (CAEU).

Organización de Estados Iberoamericanos para la Educación, la Ciencia y la Cultura (OEI) Bravo Murillo, 38.28015 - Madrid, España.

ONU. (2012). La Educación para el Desarrollo Sostenible en acción Sector Educación de la UNESCO. 7 ed., $\quad$ Paris. http://www.lacult.unesco.org/docc/2012_Educ_para_des_sost.pdf.

ONU. (2015). Sostenible, Transformar nuestro mundo: la Agenda 2030 para el Desarrollo. Obtenido de Asamblea General Naciones Unidas: https://unctad.org/system/files/official-document/ares70d1_es.pdf

Pérez, M. (2020). El desarrollo sostenible en la educación superior. Revista Científica Internacional, 3(1), 161-167. 
Pérez, E. (18 de octubre de 2018). ¿Cómo enseñar el desarrollo sostenible?: https://www.knotion.com/news/como-ensenar-el-desarrollo-sostenible

Prado, R. (2018). La socioformación: un enfoque de cambio educativo. Innovaciones educativas, 78(1), 57-82.

Sáenz, B., y De Santiago, R. (2017). "Desarrollo sostenible y educación superior en un mundo global." Revista lberoamericana de educación, vol. 73, p. 273. https://rieoei.org/historico/documentos/rie_73.pdf.

Simões, A., Yanes, G., y Alfonso, Y. (2018). Estrategia pedagógica de educación ambiental para el desarrollo sostenible en la formación del estudiante de carreras pedagógicas de Angola. Revista Universidad y Sociedad, 10 (5).

Solano, D. (2007). Desarrollo, sostenibilidad y capacidades. Una trilogía indesligable. Cuadernos de difusión, 12(23), 9-27.

Tobón, S., González, L., Salvador, J., y Vásquez, J. (2015). La Socioformación: Un Estudio Conceptual. Paradigma, 36(1), 7-29.

UNESCO - Organización de las Naciones Unidas para la Educación. (2012). Educación para el desarrollo sostenible. Libro de consulta. París: Unesco.

UNESCO (2014). Documento de posición sobre la educación después de 2015. ED14/EFA/ POST2015/1. Recuperado de http://unesdoc.unesco.org/ images/0022/002273/227336s. pdf.

UNESCO. (2019). UNESCO. Marco de aplicación de la Educación para el Desarrollo Sostenible (EDS) después de 2019. Paris: UNESCO. Conferencia General, 40th, 2019 [1185]. Obtenido de Educación para el desarrollo sostenible: https://es.unesco.org/themes/educacion-desarrollo-sostenible

UNESCO. (2021). "El Decenio de las Naciones Unidas para la EDS." UNESCO. https://es.unesco.org/themes/educacion-desarrollo-sostenible/comprenderEDS/decenio-onu. 